\title{
Rebleeding and Outcome in Patients with Symptomatic Brain Stem Cavernomas
}

\author{
Antonio Arauz ${ }^{\mathrm{a}}$ Hernán M. Patiño-Rodriguez ${ }^{\mathrm{a}}$ Mónica Chavarria-Medina ${ }^{\mathrm{a}}$ \\ Mayra Becerril $^{a}$ Gabriel Mauricio Longo $^{\text {b }}$ Edgar Nathal ${ }^{b}$ \\ a Stroke Clinic, and b Vascular Neurosurgery, Instituto Nacional de Neurología y Neurocirugía, Mexico City, Mexico
}

\begin{abstract}
Keywords
Central nervous system vascular malformations .

Cavernous · Brain Stem
\end{abstract}

\begin{abstract}
Purpose: We sought to evaluate the long-term functional outcomes and identify the potential risk factors for rebleeding in patients with brain stem cavernous malformations (BCMs) who presented with hemorrhages and were surgically or conservatively treated and prospectively monitored. Methods: From January 1990 to July 2015, we included patients with first hemorrhagic episodes secondary to single BCMs. Modified Rankin score (mRS) was used for neurological status assessment. Univariate and multivariate regression statistics were used to identify the risk factors for rebleeding. Results: A total of 99 patients with BCMs hemorrhages were included (59 [59.6\%] women, mean age $37 \pm 13$ years). As initial treatments, 37 patients (37.4\%) underwent surgery and $62(62.6 \%)$ received conservative treatment. The median follow-up was 3.33 years (interquartile range 1.16-7 years; 408.3 patient/years). The rebleeding rate by patient/year was $10 \%$ in conservatively treated patients. Deterioration was significantly more frequent in patients with rebleeding ( $p=$ $0.0001)$. At the end of the follow-up, the mRS were favorable in 49 patients (65.3\%) without rebleeding, whereas only $8(33.3 \%)$ with rebleeding evolved to favorable outcomes
\end{abstract}

$(p=0.006)$. Lesion size $>18 \mathrm{~mm}$ (hazards ratio, HR 3.34, 95\% $\mathrm{Cl} 1.54-7.26 ; p=0.0001$ ) and ventral location or crossing the brain stem's midpoint (HR 2.5, 95\% Cl 1.14-5.46; $p=0.022$ ) were associated with a major risk of rebleeding in the univariate analysis, but only a lesion $>18 \mathrm{~mm}$ remained statistically significant (HR 2.7,95\% Cl 1.2-6.21; $p=0.016)$ in the multivariate analysis. Conclusion: $A$ lesion size $>18 \mathrm{~mm}$ was the principal factor associated with hemorrhage recurrence. The overall functional outcome was good. However, significant morbidity was attributable to rebleeding.

๑) 2017 S. Karger AG, Basel

\section{Background}

Brain stem cavernous malformations (BCMs) represent $8-35 \%$ of intracranial cavernous malformations [1]. BCMs garner a particular interest because even mild or undetectable changes in the lesions can result in significant symptoms or deficits and they have an apparently higher rupture rate [2]. The brain stem location makes surgical intervention difficult without significant postoperative morbidity, and there is currently no consensus regarding the best treatment $[1,3,4]$. Series that have examined the natural history of untreated BCMs have reported wide variations in the hemorrhage rate and rebleeding [1, 5-8]. However, there is a relatively strong consensus that

\section{KARGER}

(c) 2017 S. Karger AG, Basel

E-Mail karger@karger.com

www.karger.com/ced
Hernán M. Patiño-Rodriguez, MD

Stroke Clinic, Instituto Nacional de Neurología y Neurocirugía

Insurgentes Sur 3877 Col. La Fama

CP 14269 Mexico City (Mexico)

E-Mail hemaparo@yahoo.com 
patients who present with significant neurological deficits should be surgically treated if the lesion comes to a surgical surface and patients with deep lesions are candidates for conservative treatment. Unfortunately, there are not well-defined factors for predicting the courses of individual patients or identifying patients with greater risks of possible devastating brain stem recurrent hemorrhages. Some series have identified female gender, age, larger lesions, non-lobar locations, and initial hemorrhages as risks factors for hemorrhage recurrence, but these studies have included patients with lesions along the central nervous system, which makes the results difficult to extrapolate to patients with sole BCMs $[1,2,8-12]$. The risk factors for rebleeding in patients with hemorrhagic BCMs and the long-term functional outcomes remain unclear. The main objective of our series was to evaluate the longterm functional outcomes and to identify the potential risk factors for rebleeding in a selected cohort of patients who presented with hemorrhaging and were surgically or conservatively treated and prospectively monitored.

\section{Methods}

\section{Study Population}

From January 1990 to July 2015, we included patients with a first hemorrhagic episode secondary to a confirmed single BCM who were treated at the Instituto Nacional de Neurología y Neurocirugía in Mexico City, which is a tertiary neurological center. As previously published, all the patients' data were prospectively registered in our database, which captures a standardized protocol of 186 variables, including demographics, vascular risk factors, clinical findings, routine laboratory findings, and imaging results as well as post-hospital discharge complications and functional outcomes [13]. The inception point was the diagnosis of the first brain stem hemorrhage episode due to BCM. A first episode of hemorrhage was defined as the acute or subacute onset of headache or impaired consciousness or a new focal neurological deficit attributable to the anatomic lesion of the BCM as confirmed by radiological evidence of the presence of a hemorrhage within or outside the lesion [14]. Patients with asymptomatic lesions, lesions with only a hemosiderin ring, or slowly progressive clinical courses were not considered to have hemorrhagic events.

All patients underwent MRI in the first week of the initial symptoms. At least 2 of the authors who were blinded to the treatments and outcomes of the patients assessed the size in millimeters and the location of the BCM on T1- and T2-weighted MRI images. T1 images were used for the diagnosis, measure of size, and location of BCM and to evaluate new symptoms. Gadolinium was not used routinely, and in all cases, a close correlation was established between clinic and radiological findings. For BCMs with shared locations (i.e., the mesencephalon and pons or pons and medulla), the predominant location was allocated. We also evaluated whether the BCM was ventral or in location crossing the brain stem's midpoint [3].
Standard Protocol Approvals, Registrations, and

Patient Consent

This protocol was registered and approved by our local Ethics Committee. Because of the retrospective nature of this study, we did not require written consent for the analyses of the clinical records. At the time of the procedures, written consent was obtained for all interventional procedures.

\section{Main Variables}

Treatment

The treatments were decided based on consensus with the patient and the attending physician in terms of conservative and surgical treatment. Conservative management was recommended in the following conditions: the lesion was inaccessible or small, mild symptoms occurred with rapid recovery, and the risk of surgery involved a serious compromise of neurological function. Conservative management could include the following: hospitalization, neurological deficit vigilance, intensive care unit, and nutritional support in cases of severe dysphagia. The surgical approach was recommended if the following conditions were met: mass effect was present at the moment of the hemorrhage, hemorrhage recurrence occurred, and the lesion was accessible. The time for surgery was left to the discretion of the surgeon but was preferably performed within the first 8 weeks if the patient's condition allowed it. When necessary, additional interventions, such as ventricular shunt placement, were performed.

Follow-Up

The follow-up included biannual clinical evaluations for the first year and annual follow-ups thereafter. If neurological deterioration occurred before the next clinical evaluation, the patient was instructed to seek care in our emergency department and a new MRI was performed.

Every visit consisted of a thorough physical and neurological examination that was conducted to establish the patient's clinical status. If new abnormal findings were found, a new MRI was obtained to rule out rebleeding or persistent/new lesions in the conservative and surgical groups.

If a rebleeding occurred during follow-up, the patient was eligible to receive either a first or second surgical or conservative treatment at the discretion of the patient's attending physician.

Recurrent hemorrhage was defined using the same criteria described before for the first episode of hemorrhage, but if the patient had a residual deficit of the first event, the worsening of this deficit was also included $[5,14]$.

\section{Outcome}

A recurrent hemorrhage was defined as a sudden or worsening of a clinical neurological deficit, along with evidence of new hemorrhage or magnetic resonance imaging (5.13). In all rebleeding cases, a close correlation was established between clinic and radiological findings. If a rebleeding occurred during follow-up, the patient was eligible to receive either a first or second surgical or conservative treatment at the discretion of the patient's attending physician and was censored/excluded for the further rebleeding analyses.

The primary end points were rebleeding and the functional outcomes estimated according to the modified Rankin Score (mRS). Favorable prognoses were defined as mRS 0-2, and unfavorable prognoses were defined as mRS 3-5. Moreover, these out- 
comes were assessed in person or by telephone following a preestablished interview [15] by 2 investigators who were blinded to the treatment. In the surgically treated cases, the mRS was evaluated after and before the surgeries and during the follow-up.

The prospective recurrent annual hemorrhage rate was calculated as the number of hemorrhages divided by the duration in years of the follow-up during the period of the risk of a rebleeding. This period started on the date of the first hemorrhage presentation and ended on the date of the earliest rebleeding, surgical resection, or at the last follow-up evaluation or death, whichever occurred first. We also calculated the annual cumulative risk of rebleeding.

\section{Statistical Analysis}

The frequencies of the categorical variables were calculated. For the quantitative variables, the mean or median with the SD, or interquartile range (IQR) were estimated. Univariate analyses were conducted, via the use of $\chi^{2}$ tests, Fisher exact tests, and Student $t$ tests were applied depending on the type of data being compared. The multivariate analyses related to the rebleeding risks were conducted using Cox regression analyses, which were further illustrated using the Kaplan-Meier method. Analysis of the rebleeding-free survival began on the date of the first hemorrhage presentation and ended on the date of the earliest rebleeding or the date of censoring, which occurred at the last follow-up evaluation, death, or resection. The changes in the initial and final mRS according to treatment were compared using Wilcoxon rank sum tests. All tests were 2 -sided, and $p<0.05$ was considered significant. We used the SPSS version 20.0 statistical software package. For annual cumulative risk of rebleeding, we used the actuarial method.

\section{Results}

\section{Demographic Findings}

Over a 25-year period, the 99 patients diagnosed with BCM hemorrhages were treated (59 [59.6\%] women; mean age $37 \pm 13$ years). The most common vascular risk factors were current smoking (21.2\%), hypertension (15.2\%), dyslipidemia (6.1\%), and diabetes mellitus (5.1\%). All patients presented with sudden onset of symptoms. The most common symptoms or signs were motor deficits in 54 (54.5\%) patients, VII-XII cranial nerve dysfunctions in $54(54.5 \%)$, headaches in $51(51.5 \%)$, oculomotor disturbances in 49 (49.5\%), sensitive deficits in 43 (43.4\%), cerebellar involvement (ataxia, dysmetria, or dysarthria) in $35(35.4 \%)$, mental status disturbances (drowsiness, stupor, or coma) in 17 (17.2\%), and dysphagia in $12(12.1 \%)$.

The BCMs were located in the midbrain in $32(32.3 \%)$ cases, in the pons in 53 (53.5\%), and in the medulla in 14 (14.1\%). The median lesion size was $15 \mathrm{~mm}$ (IQR 11$19 \mathrm{~mm})$. Ventral location and crossing the brain stem's midpoint were found in $40(40.4 \%)$ and venous anomalies in $12(12.1 \%)$ patients. Thirty-seven (37.4\%) patients underwent surgery and $62(62.6 \%)$ received conservative treatment as the initial treatment.

\section{Follow-Up and Rebleeding}

The median follow-up was 3.33 years (IQR 1.167 years; 408.3 patient/years). Twenty-four patients had recurrence during follow-up, of which 13 (54\%) occurred in the first 2 years. The cumulative risk of rebleeding at 1,2 , and 5 years was $12.2,13.8$, and $19.8 \%$, respectively. Patients' demographic characteristics, medical history, lesion characteristics, clinical manifestations, treatment, and prognosis with and without rebleeding are illustrated in Table 1. No differences in age and gender were found. The medical histories were similar in both groups. The pontine location was the most frequent location in the patients with rebleeding, although no locations exhibited significantly higher risk of bleeding episodes $(p=$ $0.69)$. Ventral location or crossing the brain stem's midpoint of the BCM was present in $14(58.3 \%)$ patients with rebleeding $(p=0.04)$. The size of BCM was greater in the patients with rebleeding (median $18 \mathrm{~mm}$; IQR 12.75$22.75 \mathrm{~mm}$ ) than in the patients without rebleeding (median $15 \mathrm{~mm}$; IQR 10-18 mm; $p=0.035$ ). No clinical manifestation was significantly different between the groups; however, there was a trend toward more frequent mental status disturbances in the patients with rebleeding ( $p=$ $0.064)$. Twenty (83.3\%) patients with recurrences underwent conservative treatment; meanwhile, $4(16.7 \%)$ patients underwent surgery. The hemorrhage recurrence was more frequent in the conservatively treated patients $(p=0.028)$ with rebleeding rate of patient per year being $10 \%$.

Deterioration based on the initial and final mRS was significantly more frequent in the patients with rebleeding (13 [54.2\%] vs. 10 [13.3\%]; $p=0.0001)$. At the end of the follow-up, the mRS were favorable in 49 patients (65.3\%) without rebleeding, whereas only 8 patients $(33.3 \%)$ with rebleeding evolved to favorable outcomes (mRS $0-2 ; p=0.006$ ).

\section{Risk Factors for Hemorrhage Recurrence}

After categorizing the size of the lesion, we used the median obtained in the patients with recurrence as a cutoff. The lesion size of $>18 \mathrm{~mm}$ (hazards ratio, HR 3.34, 95\% CI 1.54-7.26; $p=0.0001$ ) and ventral location plus crossing the brain stem's midpoint (HR 2.5, 95\% CI 1.14$5.46 ; p=0.022$ ) were associated with a major risk of hemorrhage recurrence in the univariate Cox regression model. However, in the multivariate Cox regression analysis, 
Table 1. Demographic characteristics, medical history, lesion characteristics, clinical manifestations, treatment, and prognosis of patients with and without rebleeding

\begin{tabular}{|c|c|c|c|}
\hline Variables & Number of rebleeding $(n=75)$ & Rebleeding $(n=24)$ & $p$ value \\
\hline Age, years, mean (SD) & $38.53(13.81))$ & $37.85(12.87)$ & $0.137^{¥}$ \\
\hline Gender, female & $47(62.7)$ & $12(50)$ & $0.341^{\Psi}$ \\
\hline Hypertension & $10(13.3)$ & $5(20.8)$ & $0.513^{\Psi}$ \\
\hline Diabetes mellitus & $3(4)$ & $2(8.3)$ & $0.592^{+}$ \\
\hline Current smoking & $16(21.3)$ & $5(20.8)$ & $1^{\Psi}$ \\
\hline Midbrain & $24(32)$ & $8(33.3)$ & $0.9^{\Psi}$ \\
\hline Pons & $41(54.7)$ & $12(50)$ & $0.69^{\Psi}$ \\
\hline Bulb & $10(13.3)$ & $4(16.7)$ & $0.73^{+}$ \\
\hline Eloquence & $26(34.7)$ & $14(58.3)$ & $0.04^{\Psi}$ \\
\hline Venous anomaly & $10(13.3)$ & $2(8.3)$ & $0.72^{+}$ \\
\hline Lesion size, mm, median (IQR) & $15(10-18)$ & $18(12.75-22.75)$ & $0.035^{*}$ \\
\hline Sensitive alteration & $32(42.7)$ & $11(45.8)$ & $0.785^{\Psi}$ \\
\hline Cerebellar involment & $27(36)$ & $8(33.3)$ & $0.812^{\Psi}$ \\
\hline Swallowing alteration & $11(14.7)$ & $1(4.2)$ & $0.28^{+}$ \\
\hline Mental status disturbance & $16(21.3)$ & $1(4.2)$ & $0.064^{+}$ \\
\hline \multicolumn{4}{|l|}{ Therapeutic method } \\
\hline Conservative treatment & $42(67.7)$ & $20(32.3)$ & $0.016^{\Psi}$ \\
\hline Surgical treatment & $33(89.2)$ & $4(10.8)$ & \\
\hline \multicolumn{4}{|l|}{ Functional outcome } \\
\hline Favorable mRS (0-2) & $49(65.3)$ & $8(33.3)$ & $0.006^{\Psi}$ \\
\hline Unfavorable mRS (3-6) & $26(34.7)$ & $16(66.7)$ & \\
\hline Better or same mRS & $65(86.7)$ & $11(45.8)$ & $0.0001^{\Psi}$ \\
\hline Worsening in $\mathrm{mRS}$ & $10(13.3)$ & $13(54.2)$ & \\
\hline Final mRS & $2.15(1.4)$ & $3.13(1.5)$ & $0.005^{¥}$ \\
\hline
\end{tabular}

Values are mean (SD), $n(\%)$, or median (IQR).

IQR, interquartile range; DM, diabetes mellitus. ${ }^{¥}$ Student $t ;{ }^{\Psi}$ chi-cuadrado; * Mann-Whitney U; ${ }^{+}$Fisher’s exact test.

only lesions $>18 \mathrm{~mm}$ remained statistically significant (HR 2.7, 95\% CI 1.2-6.21; $p=0.016$ ).

During the first 5 years of follow-up the median rebleeding-free time in patients with BCM $\leq 18 \mathrm{~mm}$ was 52.7 and 38.88 months (log-rank test: $8.65, \mathrm{df}=1 ; p=$ 0.003 ) in patients with BCM $>18 \mathrm{~mm}$ (Fig. 1). Rebleeding was present in $31.2 \%$ of the patients with $\mathrm{BCMs}>18 \mathrm{~mm}$ and $13.4 \%$ of those with BCMs $<18 \mathrm{~mm}$. Rebleeding was more frequent during the first 5 years of follow-up.

\section{Outcome According the Final Treatment}

Eleven (17.4\%) patients in the conservative group underwent surgery due to rebleeding, and $3(8 \%)$ patients in the surgical group experienced new neurological deficits and required re-interventions. At the end of the follow- up; 51 patients received conservative treatment, and 48 patients underwent surgeries. In patients who were treated with surgery, the BCM location was the midbrain in $14(29.2 \%)$, the pons in $29(60.4 \%)$, and the medulla in $5(10.4 \%)$. Figure 2 illustrates the mRS according to treatment.

In the conservative group, the initial and final mRS scores were favorable in $29(56.9 \%)$ patients. However, at the end of the follow-up, we found a higher proportion of patients with mRS of $0-1$ (18 patients [25.5\%]).

In the surgical treatment group, a pre-surgical $\mathrm{mRS}$ of $0-2$ was observed in $24(50 \%)$ patients, whereas at the end of the follow-up, favorable outcomes (mRS 0-2) were observed in $28(58.4 \%)$ patients, and in 13 patients (27.1\%), final $\mathrm{mRS}$ was 1 . 
Fig. 1. Time to rebleeding stratified by the size of the lesion.

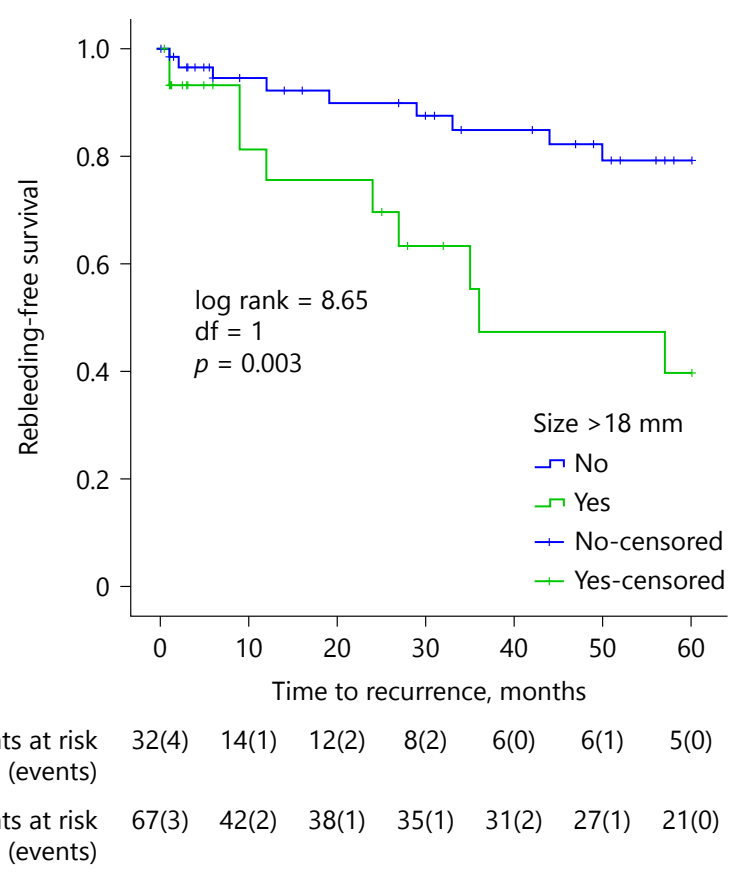

$<18 \mathrm{~mm}$ (events)

Fig. 2. mRS variation according to medical and surgical treatment.

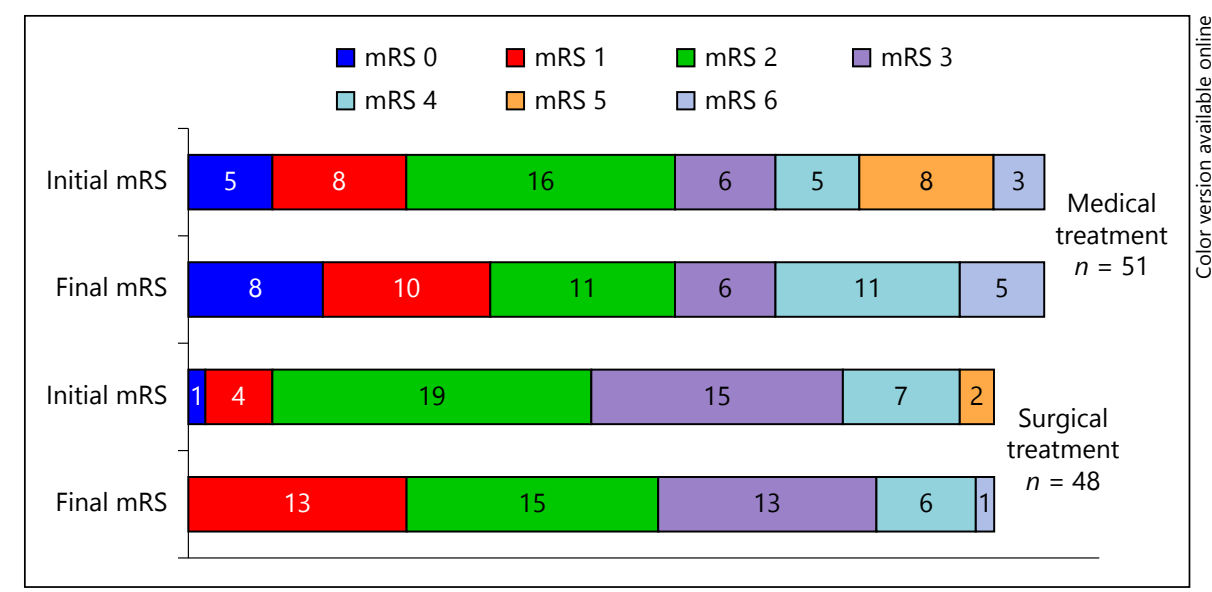

The mortality in the nonsurgical group was $9.8 \%$ (5 patients) vs. $1(2.1 \%)$ in the surgical group $(p=0.2)$.

\section{Discussion}

BCMs are generally associated with severe neurological deficits due to repeated hemorrhages. Once cavernomas bleed, the rebleeding rate increases to $45 \%$ per person per year $[3,5,16]$. The optimal treatment for these lesions remains a matter of debate, and how to identify caverno- mas that are at high risk of developing hemorrhage recurrences or producing severe neurological deficits remains unclear. The better understanding of the natural history of BMC will broaden the conservative or surgical treatment indications of these rare lesions.

In our series, the annual hemorrhage recurrence rate was higher in patients under conservative treatment (10\%) which is higher that reported in a series of patients with asymptomatic lesions $[3,5]$. We recognize that there are inherent biases in the annual hemorrhage recurrence rate calculation, since the rebleeding rate after 
a bleed are higher than in incidentally detected BCM [17, 18]. In many surgical reports, the annual re-hemorrhage rates have been variable and generally high, ranging from 15 to $60 \%$. Hauck et al. [17] and Pandey et al. [11] reported 45 and $31.5 \%$ rates, respectively. In some series, the rate of bleeding may have been overestimated because the rates were calculated based on retrospective analyses of bleed rates based on the assumption that the cavernous malformations are congenital lesions $[3,7,9$, 17]. In other series, the rebleeding rates were calculated according to the observational period, which is defined as the period from the first bleed to the time of surgery. Therefore, only patients who were surgically treated were included. In a recent meta-analysis of the natural history of untreated cerebral cavernous malformations, 575 cases of BMC were included [18]. Brain stem location was independently associated with the occurrence of intracranial cerebral hemorrhage (30.8\% 5-year risk bleed). These findings are consistent with other series and confirm that BMC have a higher rate of bleeding than cavernomas in other locations. Our study includes a highly selected cohort of patients with symptomatic lesions or with recent hemorrhage, because once they start to bleed, a second bleeding is likely to occur, and in these sites, this could be very harmful for the patient. In contrast to other reports, we calculated the rate of bleeding divided by the number of hemorrhages by the duration in years of the follow-up during the period in terms of the risk of rebleeding.

We identified the rates and predictors of recurrent hemorrhage and functional outcome. Lesions $>18 \mathrm{~mm}$ were an independent risk factor for rebleeding. This finding is consistent with those reported by other authors. Porter et al. [8], Kupersmith et al. [9] found that lesions sizes of $>10$ and $>20 \mathrm{~mm}$ are independent risk factors for rebleeding [5]. Although these are not the same lesion sizes as those considered in the current study, these findings suggest that greater extents of lesions are associated with greater risks of recurrent bleeding. Our data suggest that patients with lesions $<18 \mathrm{~mm}$ are subjected to twice the free-rebleeding time of those with larger lesions. This finding facilitates the identification of patients at higher risk of future neurological deterioration.

Another important finding of our series is that most recurrences occurred within the first 5 years, with a clear tendency to be more frequent in the first 2 years. Hauck et al. [17] reported the first recurrence in 43 $\mathrm{BCM}$ in a maximum time of 4 years, and $\mathrm{Li}$ et al. [7] reported 129 patients with recurrence of which 110
(85.3\%) occurred within the first 2 years. However, our data suggest that not all BCM values will present with hemorrhages and cause progressive neurological deficits or rebleeding during a follow-up period of 5 years. Because, at the last follow-up, $56.9 \%$ of the patients treated conservatively and $58.4 \%$ of those treated surgically had good outcomes, the differences between the initial and final $\mathrm{mRS}$ in both treatment groups was not significantly different. The outcomes were better in the patients without recurrence and rebleeding were very harmful for the patients. Seventy-seven percent of the patients with rebleeding developed severe neurological deficits.

Many studies have identified the adverse effects of female sex and an age $<40$ years $[3,9,10]$. However, our study failed to confirm these rebleeding risk factors. We found that the lesion sizes $>18 \mathrm{~mm}$ and ventral location and crossing the brain stem's midpoint were the major risk factors for hemorrhage recurrence, which is important when deciding upon the best treatment for an individual patient. Our data suggest that patients who present with hemorrhages secondary to BCM $>18 \mathrm{~mm}$ and eloquent hemorrhages should be considered for surgical treatment, although there is still the need for a prospective, randomized study.

Many data described previously by other authors have been confirmed in our series: (1) the frequent pontic localization of the lesion $(53.5 \%)$; (2) the low rate of complications and mortality among the surgical treated patients; (3) the high rate of good postoperative results; (4) the overall good functional outcome for all patients without hemorrhage recurrence; and (5) the low probability of a favorable mRS after rebleeding.

The mortality in our patients $(6.1 \%)$ was similar to the previously reported range of $0-17 \%$ [6].

The main strengths of our study include the long-term follow up in all our patients, which includes one of the greatest series of Hispanic patients with the largest follow-up that has been conducted thus far. The use of clinical and radiological criteria to confirm the presence of hemorrhage guarantees that all patients entered the study at the same time in the natural history of the disease, which helped us analyze a larger group of at-risk patients than previous published series that have included asymptomatic patients. Additionally, we used blind and standardized functional outcome evaluations that guaranteed bias-free results.

A limitation of our study is the sample size. Nevertheless, we have a 25-year cohort from a tertiary reference center, which is a sample of our local population.
Arauz/Patiño-Rodriguez/ Chavarria-Medina/Becerril/Longo/Nathal 
To our knowledge, this is the largest cohort of Hispanic people with BCM and it is probably the largest study focused on rates and predictors of recurrent hemorrhage. Although we included all patients who were admitted to our hospital, patients with first fatal events may have been missed. Other limitations include the absence of treatment group randomization; however, we did not make direct comparisons between the 2 treatments groups to avoid any possible bias and caution is advised in the interpretation of the conclusions on the efficacy of the treatments. BCMs are rare lesion and referral bias is common at centers that treat them. Finally, we did not evaluate the genotype of our patients, which is a factor that has been demonstrated can explain the different forms of expression of the severity of the disease [19].

\section{Conclusions}

Lesion sizes $>18 \mathrm{~mm}$, ventral, or in location crossing the brain stem's midpoint were the principal factors associated with hemorrhage recurrence. The overall functional outcome was good. However, significant morbidity was attributable to rebleeding.

\section{Sources of Funding}

None.

\section{Disclosure Statement}

The authors declare that they have no conflict of interest.

\section{References}

1 Moultrie F, Horne MA, Josephson CB, Hall JM, Counsell CE, Bhattacharya JJ, et al: Outcome after surgical or conservative management of cerebral cavernous malformations. Neurology 2014;83:582-589.

2 Cantu C, Murillo-Bonilla L, Arauz A, Higuera J, Padilla J, Barinagarrementeria F: Predictive factors for intracerebral hemorrhage in patients with cavernous angiomas. Neurol Res 2005;27:314-318.

3 Garcia RM, Ivan ME, Lawton MT: Brainstem cavernous malformations: surgical results in 104 patients and a proposed grading system to predict neurological outcomes. Neurosurgery 2015;76:265-277; discussion 277-278.

4 Chotai S, Qi S, Xu S: Prediction of outcomes for brainstem cavernous malformation. Clin Neurol Neurosurg 2013;115:2117-2123.

5 Li D, Hao SY, Jia GJ, Wu Z, Zhang LW, Zhang JT: Hemorrhage risks and functional outcomes of untreated brainstem cavernous malformations. J Neurosurg 2014;121:32-41.

6 Al-Shahi Salman R, Hall JM, Horne MA, Moultrie F, Josephson CB, Bhattacharya JJ, et al: Untreated clinical course of cerebral cavernous malformations: a prospective, population-based cohort study. Lancet Neurol 2012; 11:217-224.

7 Li D, Yang Y, Hao SY, Wang L, Tang J, Xiao $\mathrm{XR}$, et al: Hemorrhage risk, surgical management, and functional outcome of brainstem cavernous malformations. J Neurosurg 2013; 119:996-1008.

8 Porter PJ, Willinsky RA, Harper W, Wallace MC: Cerebral cavernous malformations: natural history and prognosis after clinical deterioration with or without hemorrhage. J Neurosurg 1997;87:190-197.

9 Kupersmith MJ, Kalish H, Epstein F, Yu G, Berenstein A, Woo H, et al: Natural history of brainstem cavernous malformations. Neurosurgery 2001;48:47-53; discussion 53-54.

10 Kondziolka D, Lunsford LD, Kestle JR: The natural history of cerebral cavernous malformations. J Neurosurg 1995;83:820-824.

11 Pandey P, Westbroek EM, Gooderham PA, Steinberg GK: Cavernous malformation of brainstem, thalamus, and basal ganglia: a series of 176 patients. Neurosurgery 2013;72: 573-589; discussion 588-589.

12 Choquet $\mathrm{H}$, Nelson J, Pawlikowska L, McCulloch CE, Akers A, Baca B, et al: Association of cardiovascular risk factors with disease severity in cerebral cavernous malformation type 1 subjects with the common Hispanic mutation. Cerebrovasc Dis 2014;37: $57-63$.

13 Arauz A, Murillo L, Márquez JM, Tamayo A, Cantú C, Roldan FJ, et al: Long-term risk of recurrent stroke in young cryptogenic stroke patients with and without patent foramen ovale. Int J Stroke 2012;7:631-634.
14 Al-Shahi Salman R, Berg MJ, Morrison L, Awad IA; Angioma Alliance Scientific Advisory Board: Hemorrhage from cavernous malformations of the brain: definition and reporting standards. Angioma Alliance Scientific Advisory Board. Stroke 2008;39:32223230.

15 Bruno A, Akinwuntan AE, Lin C, Close B, Davis $K$, Baute V, et al: Simplified modified Rankin scale questionnaire: reproducibility over the telephone and validation with quality of life. Stroke 2011;42:2276-2279.

16 Washington CW, McCoy KE, Zipfel GJ: Update on the natural history of cavernous malformations and factors predicting aggressive clinical presentation. Neurosurg Focus 2010; 29:E7.

17 Hauck EF, Barnett SL, White JA, Samson D: Symptomatic brainstem cavernomas. Neurosurgery 2009;64:61-70; discussion 70-71.

18 Horne MA, Flemming KD, Su IC, Stapf C, Jeon JP, Li D, et al: Clinical course of untreated cerebral cavernous malformations: a metaanalysis of individual patient data. Lancet Neurol 2015;pii:S1474-4422(15)00303-8.

19 Choquet H, Pawlikowska L, Nelson J, McCulloch CE, Akers A, Baca B, et al: Polymorphisms in inflammatory and immune response genes associated with cerebral cavernous malformation type 1 severity. Cerebrovasc Dis 2014;38:433-440. 